\title{
Senescence of tumor cells induced by oxaliplatin increases the efficiency of a lipid A immunotherapy via the recruitment of neutrophils
}

\author{
Cédric Seignez ${ }^{1}$, Amandine Martin ${ }^{1}$, Claire-Emmanuelle Rollet ${ }^{1}$, Cindy Racoeur ${ }^{1}$, \\ Alessandra Scagliarini ${ }^{1}$, Jean-François Jeannin ${ }^{1}$, Ali Bettaieb ${ }^{1}$, Catherine Paul ${ }^{1}$ \\ ${ }^{1}$ EPHE Cancer Immunotherapy Laboratory, EA7269 EPHE-University of burgundy, Dijon, F-21000, France \\ Correspondence to: \\ Catherine Paul, e-mail: catherine.paul@u-bourgogne.fr \\ Keywords: senescence, colorectal cancer, immunotherapy associated chemotherapy, neutrophil \\ Received: August 07, 2014 \\ Accepted: September 30, 2014 \\ Published: October 16, 2014
}

\section{ABSTRACT}

Management of advanced colorectal cancer is challenging due to the lack of efficient therapy. The lipid A, OM-174 exhibited antitumor activity in colorectal cancer. We explored the anticancer efficacy of this compound in rats bearing large colorectal tumors in combination with the platinum derivative drugs oxaliplatin and cisplatin. While each drug used alone exhibited partial antitumor activity, sequential treatment with oxaliplatin or cisplatin for one week followed by lipid A injections induced a great regression of colorectal tumors, with more than $\mathbf{9 5 \%}$ of rats cured from their tumors. This potent antitumor efficacy of the combined treatments was correlated to the sequential induction of cellular senescence by oxaliplatin, and of apoptosis, mainly triggered by the lipid $\mathbf{A}$. Moreover, a recruitment of Tumor-Associated Neutrophils with N1 phenotype as attested by the expression of inducible nitric oxide synthase was observed with combination of oxaliplatin and lipid A. Neutrophil recruitment within tumor microenvironment was due to oxaliplatin and lipid A-dependent release of neutrophil specific chemoattractants such as cxcl1 and 2. However the $\mathrm{N} 1$ phenotype is only dependent of lipid A treatment. These results suggest that the combination of chemotherapy with an immunotherapy is a promising approach to treat patients with advanced colorectal cancer.

\section{INTRODUCTION}

Colorectal cancer is the second leading cause of death in Northern countries [1]. Despite significant developments in the treatment of this disease by using new cytotoxic chemotherapies and novel biological agents, it still causes considerable mortality particularly in patients with metastatic colorectal cancer (mCRC) [2]. Several drugs used as single agent or in various combinations are available to treat $\mathrm{mCRC}$, including fluoropyrimidines (5FU, capecitabine), irinotecan, oxaliplatin, the vascular endothelial growth factor (VEGF) antibody bevacizumab, the epidermal growth factor receptor (EGFR) antibodies cetuximab and panitumumab, and others [3]. The antitumor efficacy of these drugs has been ascribed to their ability to block the growth of tumors through different mechanisms. They can induce apoptosis of tumor cells by the activation of series of cysteine proteases called caspases [4] or their senescence, a process leading to irreversible arrest of cell division. Cells can undergo senescence through three separate pathways [5]: (i) replicative senescence, induced through shortening of telomere; (ii) stress-induced premature senescence, triggered by cellular stress, such as elevated oxygen levels or cytotoxic agents leading to extensive DNA damage; and (iii) overexpression or hyperactivation of oncogenes, such as Ras, c-myc, or BRAF. The activation of any of these senescence pathways triggers a permanent arrest of growth of transformed cells and thus prevents carcinogenesis. Even though senescent cells cannot proliferate they are still metabolically active and release a broad variety of cytokines and chemokines that modify the microenvironment.

Despite the improvement of overall survival of patients with mCRC treated with these cytotoxic chemotherapies and biological agents, the development 
of resistances has been observed. Introduction of new treatment regimens is thus required to maximize therapeutic impact and tailor the toxicity profile in patients suffering from mCRC. Significant progress in understanding the tumor-induced immune suppression mechanisms allows the development of new immunological therapeutic strategies. To date the most effective immunological anti-tumor responses have been observed with active nonspecific approaches, such as Bacillus Calmette-Guérin (BCG) [6]. Moreover, recent data demonstrate that the immune infiltrates of the tumor such as T-lymphocytes are determinant for the outcome of patients bearing a solid cancer [7]. This suggests that patient prognosis can be estimated by the evaluation of the immune component of the tumor microenvironment. Furthermore, there is growing evidence that combination of conventional chemotherapy with immunomodulation strategies is of critical importance for efficient tumor eradication [8].

We have previously shown that an analog of lipid A, OM-174 (the active component of lipopolysaccharides) exerts, in experimental models, antitumor effect against different tumor types including colon, breast cancers and melanoma $[9,10]$. In a model of peritoneal carcinomatosis induced in rats by intraperitoneal injection of syngeneic colon cancer cells, administration of lipid A induced complete regression of tumors and hemorrhagic ascites in $95 \%$ of cases [9]. This antitumor activity was associated with inflammatory cytokine secretion and inducible nitric oxide synthase (iNOS) activation [11]. However, lipid A, which safety and tolerance have been demonstrated in a phase I clinical trial in patients suffering from cancer [12], was not effective in rats bearing large tumors (21 days post-injection of cancer cells).

In the current study, we investigated the antitumor activity of platinum derivative oxaliplatin and cisplatin in combination with this analogue of lipid A in large peritoneal carcinomatosis. Moreover, we explored the mechanism of their action in vitro and in vivo, by studying their effects on apoptosis and senescence of tumor cells, and by assessing their ability to promote the recruitment of immune cells within tumors. We have shown that platinum derivatives induce tumor cell senescence which, when combined to lipid A, allows the recruitment of antitumoral neutrophils (N1), characterized by iNOS expression, within the tumors. Our findings indicate that combination of platinum derivative with lipid A is highly promising for the treatment of advanced colorectal tumors.

\section{RESULTS}

\section{Association of platinum derivatives with an analogue of lipid A eradicated large colorectal tumors in syngeneic rats}

BD IX rats bearing large syngeneic tumors due to PROb colon cancer cells (21 days after cell inoculation in rats) were randomized into 6 groups: control (vehicle), oxaliplatin or cisplatin ( $3 \mathrm{mg} / \mathrm{kg})$, lipid A (1 mg/kg), and the combination of lipid A with each platinum derivative. Treatment with one dose of oxaliplatin or cisplatin was started at day 21 post-tumor cell injection. One week later, the rats were treated with lipid A three times per week, during five weeks. Figure $1 \mathrm{~A}$ shows that the combination increased drastically the life span of rats since $95 \%$ of rats treated with oxaliplatin and lipid A were alive on day 150 and were cured of their affection (data not shown), while only $33-40 \%$ of rats treated with either lipid A or oxaliplatin and $3 \%$ of untreated rats were still alive at the same date. Furthermore, on day 70 , all the rats of the control group or treated with cisplatin only were dead while all the rats treated with the combination cisplatin and lipid A were alive (Figure 1B). We also confirmed the antitumor efficacy of oxaliplatin and lipid A in the syngenic colon cancer CT26 mouse model. As shown in Figure $1 \mathrm{C}, 50 \%$ of mice were alive after 45 days of their treatment with oxaliplatin and lipid A while nearly all mice were died when left untreated or treated with drugs when taken alone. These results indicate that sequential treatment with platinum derivative and lipid A increased the life span of rats and mice bearing large tumors.

\section{Lipid A induced apoptosis of cancer cells which was increased by oxaliplatin}

We have explored the mechanisms involved in the antitumor efficacy of platinum derivative/lipid A combination. We analyzed the ability of lipid A and oxaliplatin to induce apoptosis in tumor (TUNEL staining) and more precisely in tumor cells (M30 staining) (Figure 2A and B). The lipid A induced apoptosis in tumors after $24 \mathrm{~h}$ time period treatment of rats. Oxaliplatin had little effect on this type of cell death induction but increased the rate of apoptosis induced by lipid A. Our results also indicated that triggering and increasing of apoptosis by lipid A and oxaliplatin, respectively, were correlated to caspase- 3 activation as attested by the elevated rate of $\mathrm{M} 30$, the caspase 3-dependent cleaved form of cytokeratin 18 .

\section{Oxaliplatin induced cellular senescence in colon cancer cell lines}

We also examined the ability of lipid $\mathrm{A}$ and oxaliplatin to induce senescence in the rat $\mathrm{PROb}$ and mouse CT26 colon cancer cells. The senescenceassociated $\beta$-galactosidase (SA- $\beta$-Gal) activity in cells was determined by cytochemical detection using the $\mathrm{X}$-Gal substrate or by fluorescence detection using the DDAOG substrate [13]. Lipid A did not trigger senescence either in PROb (Fig 3A) or in CT26 (data not shown) tumors. However, oxaliplatin induced senescence into 


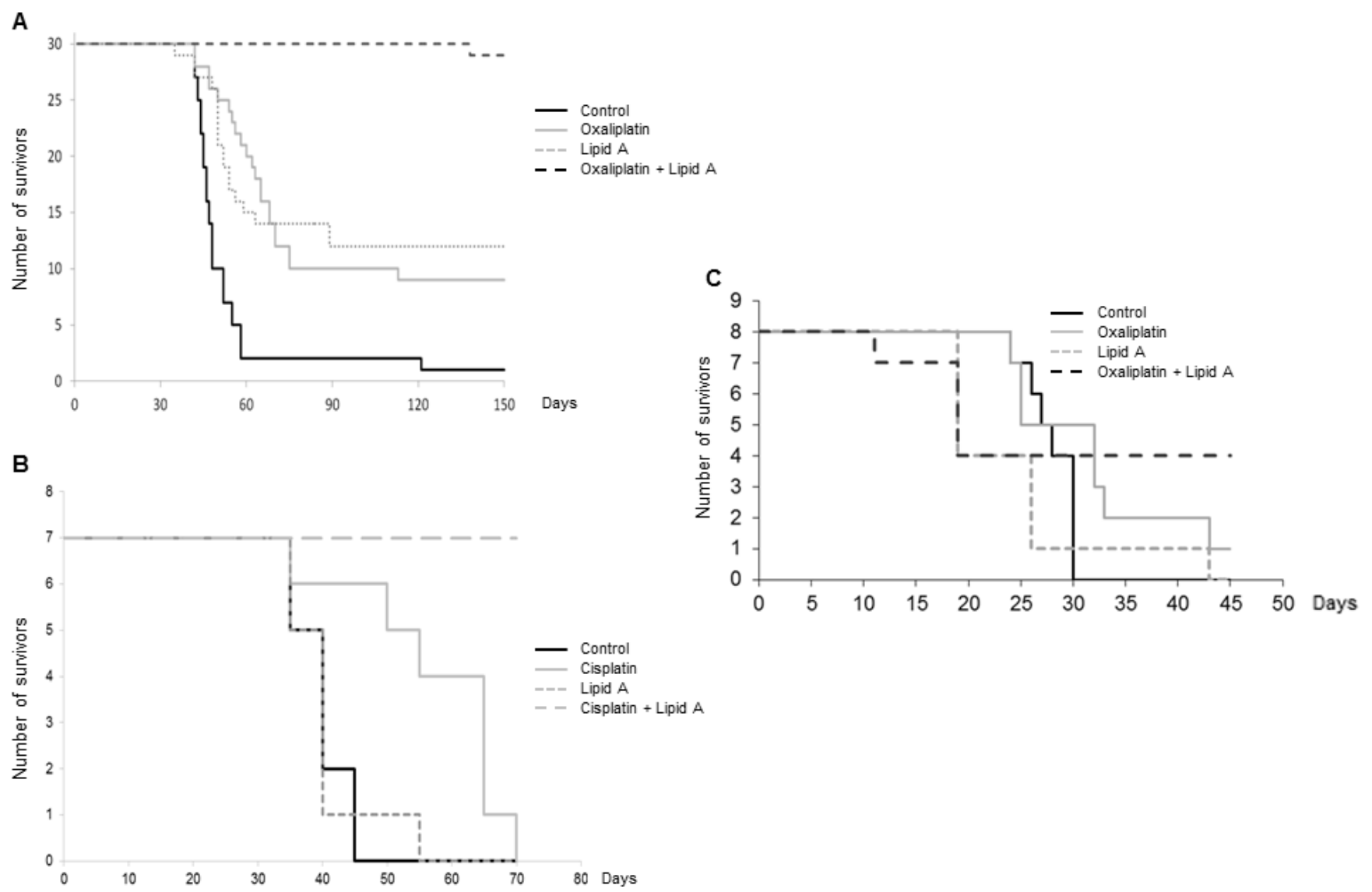

Figure 1: Oxaliplatin or cisplatin in combination with lipid A increased the lifespan of tumor-bearing rats or mice. Tumor-bearing rats were treated by oxaliplatin/lipid A (A) or cisplatin/lipid A (B) combination. Twenty-one days after i.p. injection of $10^{6} \mathrm{PROb}$ cells, rats were treated by oxaliplatin or cisplatin or saline solution (control). At twenty eight days, rats were treated with a lipid A or saline solution 3 times per week for 5 weeks. Figure A represented data from 3 independent experiments. Figure B was representative of 3 experiments with 7 animals per group. (C) Five days after i.p. injection of CT26 cells, mice were treated by oxaliplatin or saline solution (control). At ten days, mice were treated with a lipid A or saline solution five times, every five days.

the PROb cells in vivo (Figure $3 \mathrm{~A}$ and $\mathrm{B}$ ) and in the two cell lines, $\mathrm{PROb}$ and CT26, in vitro (Figure $3 \mathrm{C}$ and $\mathrm{D}$ ). Results obtained with X-Gal staining (Figure $3 \mathrm{~A}$ and $\mathrm{C}$ ) were confirmed with DDAOG staining, another method to detect cellular senescence, visualized by epifluorescence microscopy (Figure 3B) or by flow cytometry analysis (Figure 3D). It is worthy to note that senescence in tumors occurred in cancer cells as some epithelial cells (cytokeratin positive cells) became senescent after oxaliplatin treatment (Figure 3B). Oxaliplatinmediated cellular senescence was also characterized by the analysis of senescent-associated gene expression (SASP). As shown in Figure 4A, in rats bearing PROb tumors, oxaliplatin induced the expression, at the protein level, of interferon gamma (IFN $\gamma$ ), interleukin(IL)-1 $\beta$ and tumor necrosis factor (TNF) $\alpha$ (Figure 4A). This expression occurred $24 \mathrm{~h}$ after treatment and persisted at least 9 days. Some other SASP have been induced by oxaliplatin at the level of mRNA such as IL6, IL8 and matrix metalloproteinase (MMP) 3 (Figure 4B). These results indicate that oxaliplatin but not lipid $\mathrm{A}$ is a potent inducer of senescence in vivo.

\section{Oxaliplatin induced chemokines production and recruitment of $\mathrm{N} 1$ tumor-associated neutrophils}

Since senescence has been often associated with pro-inflammatory status, we analyzed the ability of oxaliplatin to induce an increased expression of cytokines in blood of tumor-bearing rats. Using a cytokine array, we determined that treatment of rats with oxaliplatin for eight days induced the high expression of CXCL1 and IL-8, two chemokines involved in the recruitment of some immune cells, particularly the neutrophils (Figure 5A). Determination of neutrophil-specific chemokines expression by RT-PCR confirmed that oxaliplatin increased cxcll, cxcl2 and il-8 gene expression in colon tumors (Figure 5B). Immunohistostaining techniques and 
A
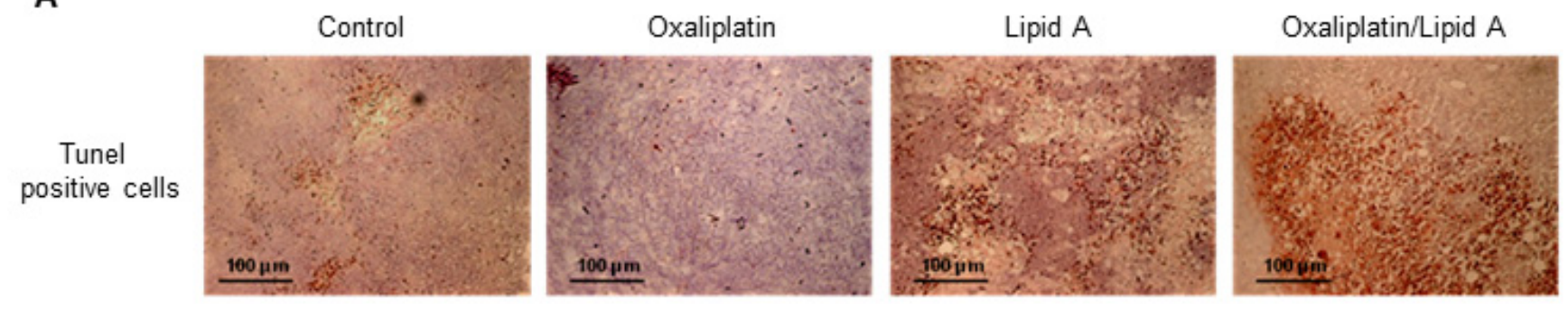

B
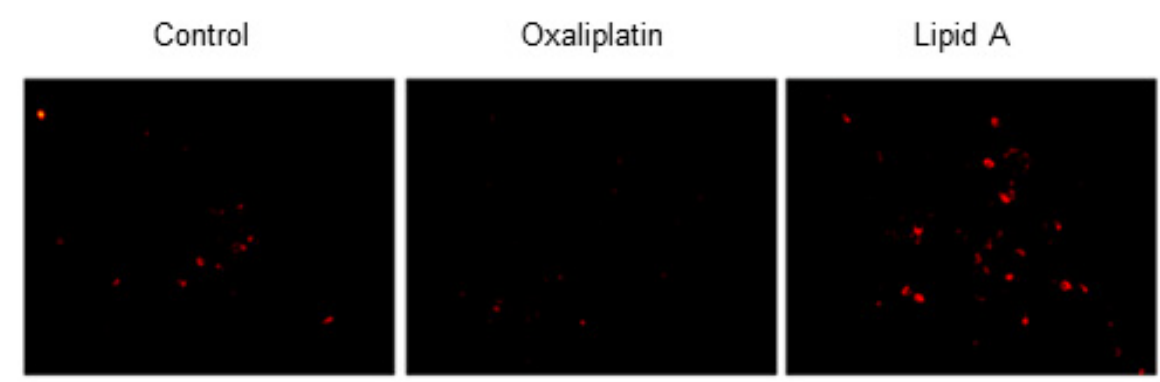

Oxaliplatin/Lipid A

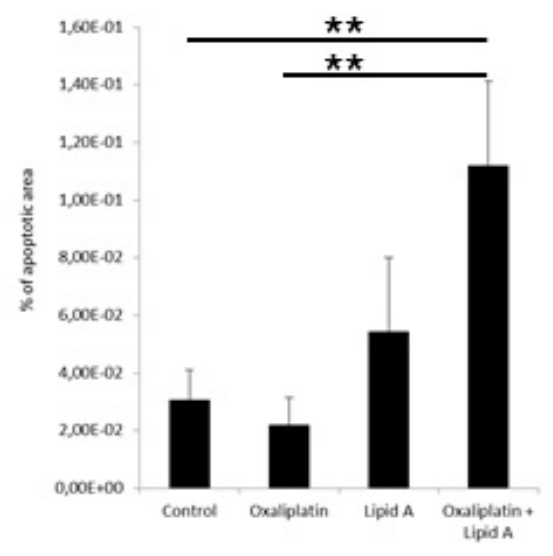

Figure 2: Lipid A induced apoptosis in tumor cells in vivo, increased by oxaliplatin. Tumor-bearing rats were treated at day 21 with oxaliplatin or saline solution (control). At day 28, rats were treated with lipid A or saline solutions. Tumors were harvested at day 29. (A) Apoptosis in tumors was detected by TUNEL staining (brown staining, upper panel). (B) Apoptosis in tumor cells was detected by M30 immunostaining (red staining, lower panel). Images are representative of 3 independent experiments with 3 animals per group. Percentage of apoptotic area on slides was represented in the lower graph. Significant differences were determined by an Anova followed by a Bonferroni test., ${ }^{* *} p<0.01$.

immunohistofluorescence showed that treatment of rats with oxaliplatin for 1 to 4 days triggered the recruitment of neutrophils into tumor microenvironment as attested by the expression of HIS-48, a specific plasma membrane antigen of rat neutrophils (Figure 5C). Neutrophils recruitment was confirmed by the increased expression of neutrophil cytolytic factor 1 (ncf1) and 2 (ncf2), two components of phagocyte NADPH oxidase expressed by neutrophils (Figure 5D). The density of neutrophils was higher inside the tumors from rats treated with the combination oxaliplatin and lipid A than from rats treated with oxaliplatin or lipid A alone (Figure 5E). The neutrophil infiltration is maintained during the lipid A treatment, at least until day 43 (data not shown). Interestingly, lipid A but not oxaliplatin induced the expression of inducible nitric oxide synthase (iNOS) in a fraction of tumorassociated neutrophils (Figure 5E). These results indicated that oxaliplatin induced the recruitment of neutrophils into tumors through the induction of neutrophil specific chemokines. In addition, a part of these neutrophils exhibited a lipid A-dependent expression of an antitumor neutrophil phenotype (N1) that may participate to the antitumor efficacy of oxaliplatin and lipid A. Neutrophils were not the only leucocytes that infiltrated tumor microenvironment. We also observed the recruitment of macrophages and lymphoid cells but at a less extend than neutrophils and occurred later in the case of lymphocytes, 9 days after oxaliplatin treatment (data not shown). 
A

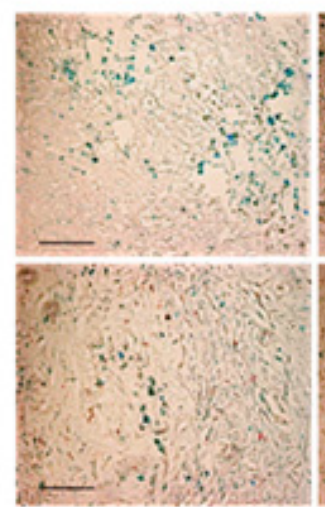

Lipid A

C

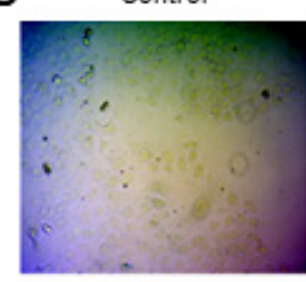

CT26 cells

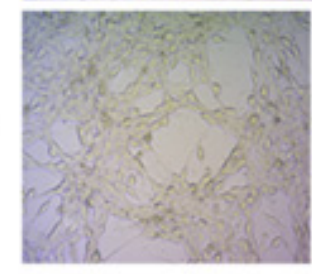

Oxaliplatin

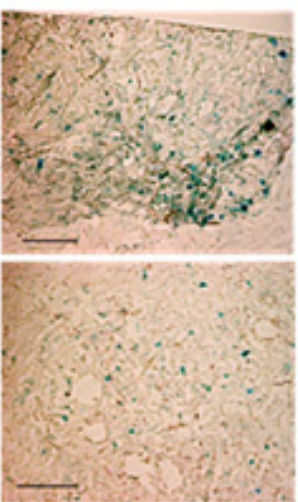

Oxaliplatin/Lipid A
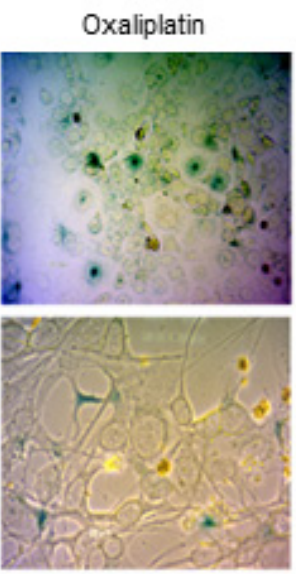

B

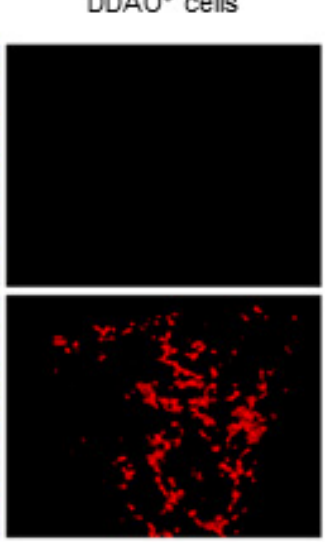

Control

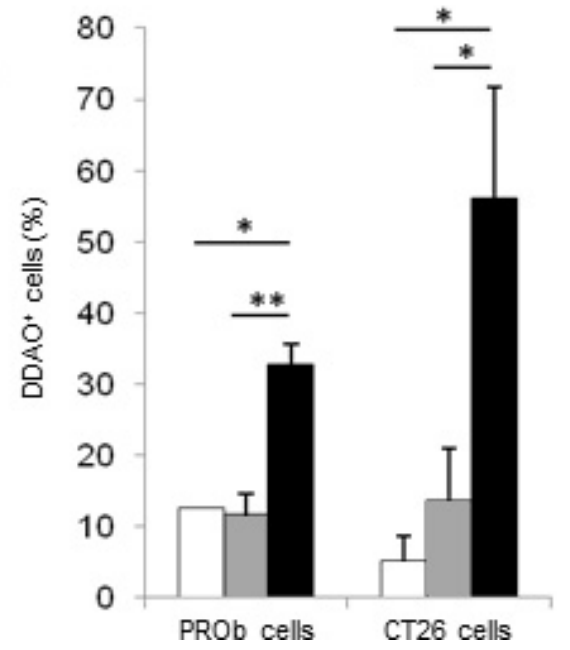

Oxaliplatin

- ControlD0

- ControlD8

- Oxaliplatin D8

Figure 3: Oxaliplatin induced senescence of tumor cells in vivo and in vitro. Oxaliplatin induced PROb cell senescence in tumors (A-B). Tumor-bearing rats were treated by oxalipatin or saline solution (control) at day 21. (A) X-Gal staining (blue) of senescent cells in tumors at day 29 (black bar, $100 \mu \mathrm{m}$ ). (B) Staining of tumor cells (anti-cytokeratin Ab, blue) and senescent cells (DDAO, red) in tumors at day 29. Images are representative of 3 independent experiments with 3 rats per group. Oxaliplatin induced tumor cell senescence in vitro (C-D). PROb or CT26 cells were treated for 5 days with oxaliplatin. (C) Senescent cells in culture were visualized by X-Gal staining (blue). (D) Quantification of $\mathrm{DDAO}^{+}$cells by flow cytometry 8 days after treatment by oxaliplatin. Bars are means \pm SEM Significant differences were determined by Mann-Whitney $\mathrm{U}$ test. ${ }^{*} p<0.05,{ }^{* *} p<0.01$.

\section{DISCUSSION}

The life span of rats bearing large nodules of colon cancer was greatly increased when animals were treated with the platinum derivative drugs cisplatin or oxaliplatin, together with an analog of lipid A. When treated animals were submitted to autopsy, most of them were free of nodules. This observation demonstrates that the treatment induced the regression of large peritoneal tumor nodules. Indeed, peritoneal carcinomatosis on the one hand and large colon cancer nodules on the other hand are associated with poor clinical outcome [14]. Thus the antitumor efficacy of these drugs combination arises as a new, promising strategy in the fight against cancer progression. In earlier studies, this lipid A analog was shown to induce tumor regression and the recovery of rats bearing macroscopic peritoneal carcinomatosis of colon cancer when treatment started on day 14 post-cell inoculation. It was shown that the treatment with lipid A analog alone induced iNOS expression and tumor cell apoptosis within 24 hours $[9,11,15]$. Here, we show that the antitumor efficacy of the lipid A analog alone was strongly decreased when the treatment started later, i.e. on day 28 post-cell inoculation (Figure 1). However, lipid A maintained its ability to induce tumor cell apoptosis in large tumors sensitized by platinum derivatives (Figure 2). It has been reported for some cancers that TLR4 agonists represent promising vaccine adjuvants on the one hand and are successfully and safely used in classical monotherapy on the other hand. Indeed, clinical benefit was observed in a randomized Phase III trial after vaccinating CRC patients with autologous tumor cells and BCG as adjuvant [16]. Monophosphoryl lipid A is also used as vaccine adjuvant with excellent safety and immunogenicity in several cancers [17, 18]. Additional TLR2/TLR4 agonists such as picibanil have been shown to be well tolerated and 

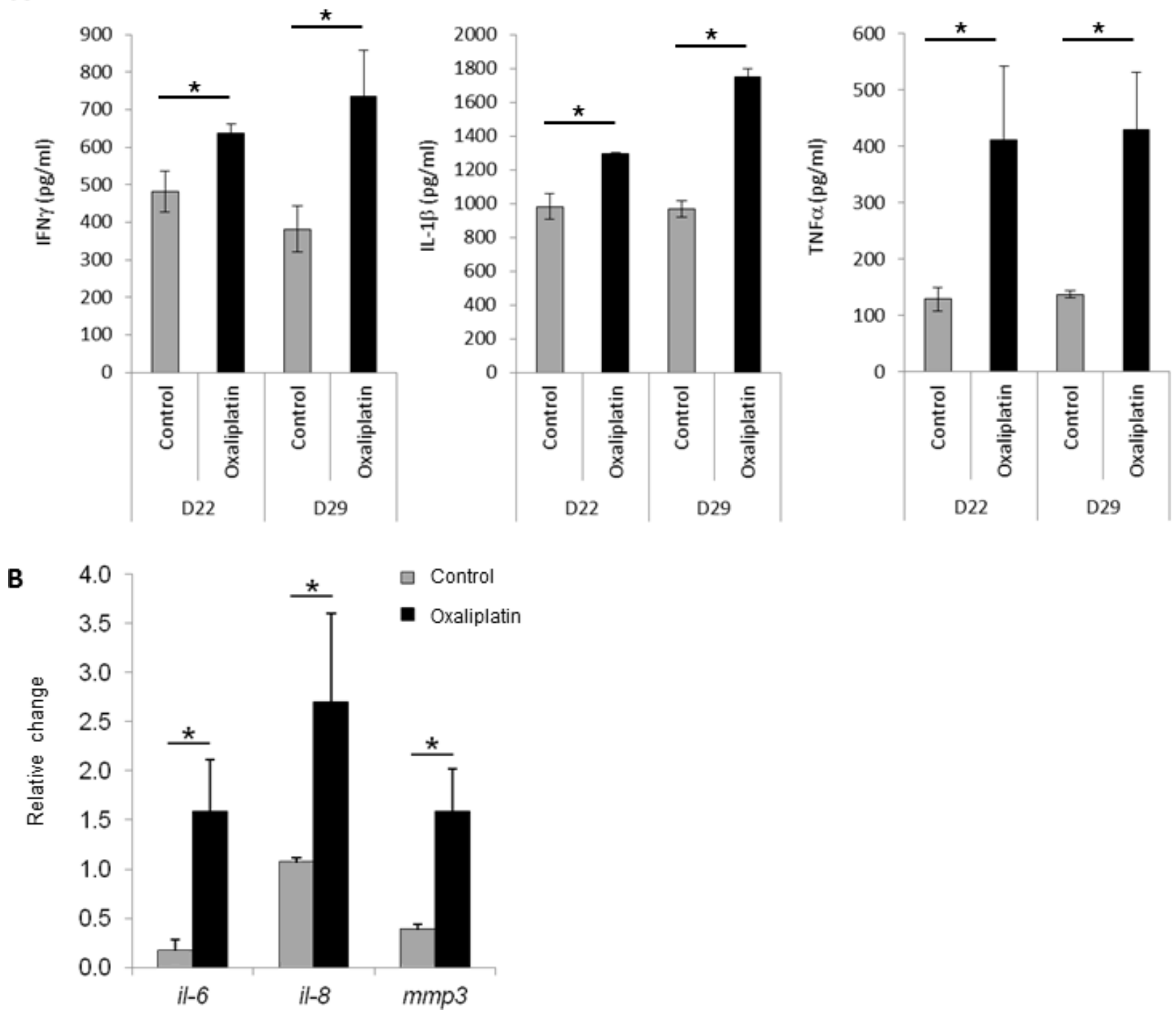

Figure 4: Expression of senescence-associated genes after oxaliplatin treatment in vivo and in vitro. Tumor bearing rats were treated with oxaliplatin or saline solution (control) at day 21. (A) At days 22 or 29 , tumors were harvested and IFN- $\gamma$, IL-1 $\beta$ and TNF- $\alpha$ concentrations were determined by ELISA. (B) Ninety six hours later injection, tumors were harvested and expression level of $i l-6, i l-8$ and mmp 3 mRNAs was determined by RT-PCR and normalized to gapdh gene expression. Bars are means \pm SEM. Significant differences were determined by Mann-Whitney U test. ${ }^{*} p<0.05$.

effective as an adjuvant to cisplatin in a cohort of patients with malignant pleural effusions [19]. Moreover, our team also performed a phase I clinical trial with the synthetic lipid A OM-174 in which its safety and tolerance was demonstrated in patients suffering from cancer [12].

Our results also showed that although lipid A induced cancer cell apoptosis, it failed to increase cellular senescence, contrarily to oxaliplatin which did not act on apoptosis but was a potent inducer of cellular senescence. Even though we did not demonstrate the involvement of apoptosis and senescence in tumor regression, one can speculate that these two pathways should contribute to the antitumor efficacy of oxaliplatin and lipid A, since abundant evidence points to a crucial physiological role for apoptosis and senescence in counteracting tumorigenesis. Recent studies reported that chemotherapyinduced senescence is one of key determinants of tumor response to therapy. For example, cisplatin has been demonstrated to induce cellular senescence in various cancer cells [20, 21]. Oxaliplatin also induced cell senescence in hepatocellular carcinoma cell lines [22]. To our knowledge, there are no data in the literature about the ability of oxaliplatin to induce senescence in colon cancer cells. It was recently reported that 5-FU/leucovorin-treated 
A
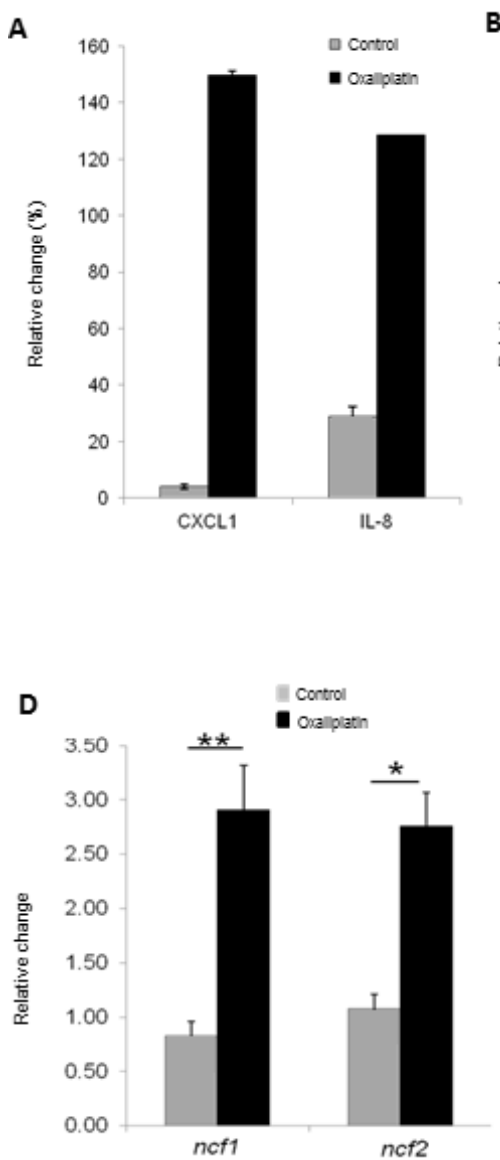

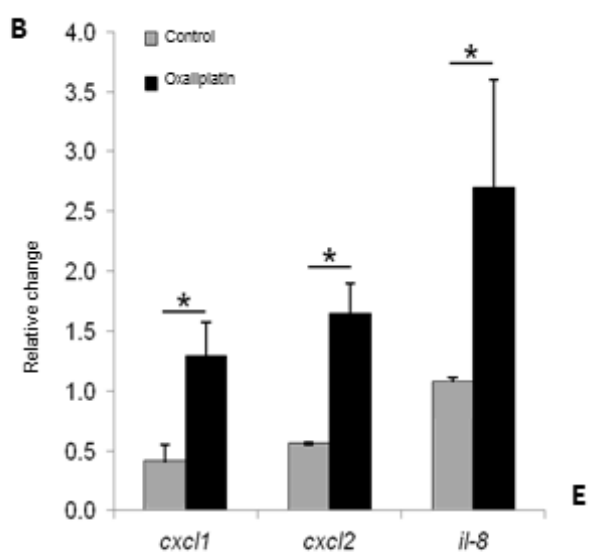

Control

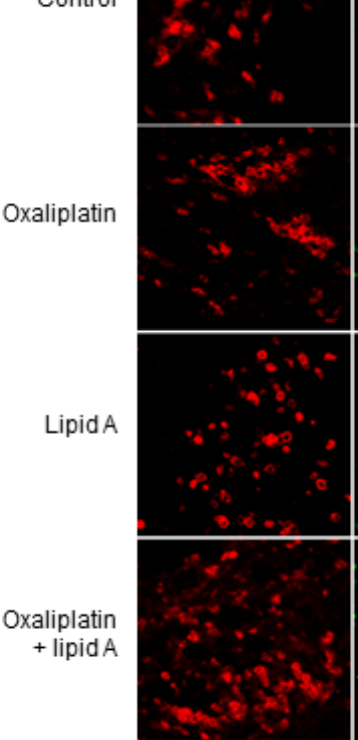

D22
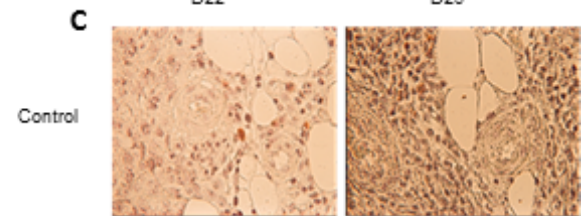

Oxaliplatin
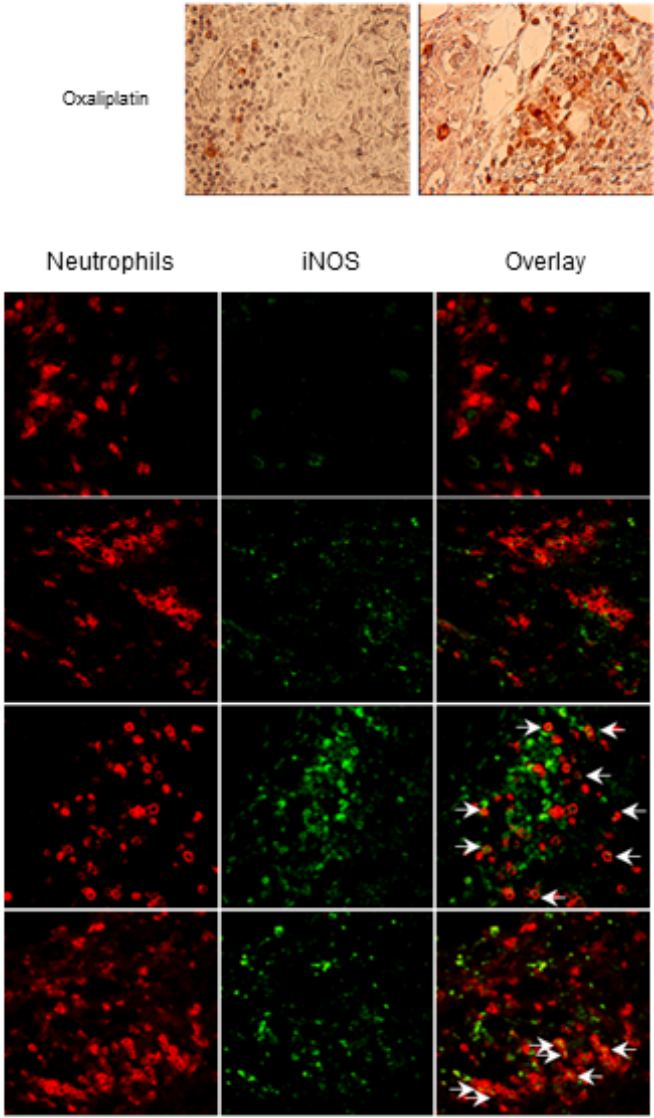

Figure 5: Oxaliplatin induced chemokine production which associated with lipid A, induced the iNOS-expressingneutrophil recruitment. (A) Eight days after injection of oxaliplatin or saline solution (control), blood was collected and plasma level of chemokines was analysed by cytokine array. The relative abundance of CXCL1 and IL-8 was expressed as a percentage of an internal control. (B) Tumor-bearing rats were treated by oxaliplatin or saline solution (control) at day 21. Ninety six hours later, tumors were harvested and expression levels of $c x c l 1, c x c l 2$ and $I l-8$ mRNAs were determined by RT-PCR and normalized to gapdh gene expression. (C) The recruitment of neutrophils in tumors from control or oxaliplatin-treated rats was determined by immunostaining (anti-HIS48 Ab, red) of tumors harvested at day 22 and 25. (D) As in (B), tumors were harvested and RNA was extracted. Expression levels of $n c f 1$ and $n c f 2$ were determined by RT-PCR and normalized to gapdh gene expression. Bars are means \pm SEM. Significant differences were determined by Mann-Whitney U test. ${ }^{*} p<0.05,{ }^{* *} p<0.01$. (E) Tumors from control, oxaliplatin, lipid A or oxaliplatin + lipid A rats were removed at day 29, fixed, cut into 5-mm cryosections and stained for neutrophil (anti-HIS48 Ab, red) and iNOS (anti-iNOS Ab, green). Merged signals (yellow) are pointed with white arrows in the overlay pictures. (C and $\mathbf{E})$ are representatives of 3 independent experiments with 3 animals per group (scale bars $=50 \mu \mathrm{m}$ ).

CRC patients achieved a significantly longer progression free survival when presenting with senescence indexpositive tumors before treatment [23]. Based on these data, we can speculate that CRC patients with positive senescence index may represent a cohort of patients with a favorable outcome when treated with lipid A or conversely that CRC patients with negative senescence index may represent good candidates for oxaliplatin/lipid A treatment.

Even though we did not analyze the molecular mechanisms by which oxaliplatin induced cellular senescence, several mechanisms have been reported in the literature. Recent evidence emerges that the mammalian target of rapamycine (mTOR) pathway is involved in cellular senescence [24]. Indeed, inhibition of this pathway, for example, by hypoxia [25], by contact inhibition or by high cell density [26] suppresses senescence program. Further, reactivation of mTOR in quiescent cells causes senescence after treatment with DNA damaging agents [27]. In our case, mTOR activation could be at the origin of oxaliplatin-mediated cellular 
senescence. Recently, it has been reported that treatment of cholangiocarcinoma cells with oxaliplatin increased the phosphorylation of mTOR [28].

Our results also showed that the antitumor efficacy of oxaliplatin and lipid A was correlated to tumor infiltration of neutrophils. This recruitment within tumors could be due to the induction of cellular senescence by oxaliplatin. Accordingly, it has been observed infiltration of neutrophils, macrophages and NK cells in regressing tumor triggered by p53 activation and thus induction of cellular senescence [29]. Some of these neutrophils expressed iNOS, one hallmark of N1 phenotype of neutrophils. These cells in tumor may have differential states of activation/differentiation, thus taking an antitumorigenic ("N1") versus a protumorigenic ("N2") phenotype [30]. Interestingly, the acquisition of the N1 phenotype after lipid A treatment was also found in neutrophils from bone marrow and spleen (data not shown). Thus, our data show that activation with lipid A could promote the conversion from tumor-promoting to tumor-suppressing neutrophils which could participate to the antitumor efficacy of oxaliplatin and lipid A.

In summary this study describes a new therapeutical strategy to eradicate advanced colon cancers by combining chemotherapeutic agents such as platinum derivatives with immunomodulators such as lipid A analogs. This association is linked with the induction of senescence and apoptosis of tumor cells in vivo and with the recruitment of neutrophils with an N1 phenotype.

\section{METHODS}

\section{Cell culture}

$\mathrm{PROb}$ colon cancer cells were harvested from a colon carcinoma induced in BD-IX rat [31]. CT26 murine colon cancer cells were obtained from the ATCC. PROb and CT26 cells were maintained at $37^{\circ} \mathrm{C}$ in HAM's F10 and RPMI medium, respectively, supplemented with $10 \%$ FBS (Lonza).

\section{Animal tumor models and treatments}

Male BD-IX rats (2-3 month-old) were purchased from Charles River (L'arbresle, France). Carcinomatosis in BD-IX rats was induced by i.p. injection of $1.10^{6}$ colon cancer PROb cells as previously described [9]. Oxaliplatin or cisplatin treatment $(3 \mathrm{mg} / \mathrm{kg}$, single i.p. injection) was performed 21 days later; control treatment consisted of saline injection. Immunotherapy treatment began 28 days after carcinomatosis induction and consisted of 15 injections of lipid A (1 mg/kg, i.v. injections) or of saline solution for controls over a 5 -week period. Carcinomatosis in Balb/C mice was induced by i.p. injection of $5.10^{5} \mathrm{CT} 26$ cells. Oxaliplatin treatment $(3 \mathrm{mg} / \mathrm{kg}$, single i.p. injection) was performed 5 days later; control treatment consisted of saline injection. Immunotherapy treatment began
10 days after carcinomatosis induction and consisted of 5 injections of lipid A ( $8 \mathrm{mg} / \mathrm{kg}$, i.v. injections) or of saline solution for controls over a 20-day period. All animal procedures were approved by the Ethics Committee of the University of Burgundy (protocols 2006 and 3904).

\section{Antibodies and reagents}

Mouse anti-HIS48 antibody (Ab) (Santa Cruz) was used to characterize tumor-infiltrating rat neutrophils. Tumor cells were identified using a mouse anti-cytokeratin antibody (DakoCytomation). The following Abs were also used: a rabbit anti-iNOS Ab (BD Transduction Laboratories) and a mouse anti-M30 fragment (Roche). Secondary anti-mouse or anti-rabbit Alexa Fluor 568 or 488 conjugates antibodies were purchased from Molecular Probes, Invitrogen. X-Gal (Roche) and DDAOG (Life Technologies) were used to characterize SenescenceAssociated- $\beta$-Galactosidase (SA- $\beta$-Gal) activity into the cells. The lipid A analog OM-174 was kindly provided by OM Pharma (Meyrin, Switzerland).

\section{Detection of cytokines by Ab array}

Blood was collected by heart puncture under isoflurane anesthesia. The same volume of plasma was applied onto antibody-coated membranes for rat cytokine array (R\&D Systems) according to the manufacturer's protocol. Arrays were scanned as digital images and signal intensity was quantified with ImageJ software.

\section{Detection of cytokines by ELISA}

IFN- $\gamma$, Il- $1 \beta$ and TNF- $\alpha$ concentrations in tumor lysates were detected by ELISA (Uscn Life Science Inc) according to the manufacturer's protocol. Absorbance was read on a spectrophotometer (Asys UVM 340) at $450 \mathrm{~nm}$ wavelength.

\section{RT-PCR}

Total RNA was extracted from tumors and cell lines using the TRIzol reagent (Gibco-BRL) according to the manufacter's instructions. RT-PCRs were performed with the Qiagen OneStep RT-PCR kit (Qiagen) according to the manufacturer's protocol. The rat primers used (Invitrogen) were the following: $\mathrm{cxcll}$ (f) 5'-GAGAAAGAAGATAGATTGCACCGATG-3' and (r) 5'-TTCTTCCCGCTCAACACCTTC-3'; cxcl2 (f) 5'-GTGACACTGAAGAGTTACGATGTCAG-3' and (r) 5'- CCTGAGGCTCCATAAATGAAAGA-3'; ncfl (f) 5'-CACCGAGATCTACGAGTTCC-3' and (r) 5'-TCCCATGAGGCTGTTGAAGTAC-3'; $n c f 2$ (f) 5'-GAAAGCATGAAGGATGCCTGG-3' and (r) 5'- ATAGCACCAAGATCACATCTCCTTCC-3'; il-6 (f) 5'- TTCTCTCCGCAAGAGACTTCCAGCC-3' and (r) 5'- AAACGGAACTCCAGAAGACCAGAGC-3'; $i l-8$ (f) 
5'- AGCGGTTCCATCTCGCCATTCATGC-3' and (r) 5'- TGAACACTGGCCGTTCTTTCCACTGC-3'; mmp3 (f) 5'- GTACGGCTGTGTGCTCATCCTACC-3' and (r) 5'- AAGTCTCCATGTTCTTCAACTGCAAAGG-3'. To standardize the cDNA samples, expression of the housekeeping gene gapdh was tested with primer pair (f) 5'-GGCACAGTCAAGGCTGAGAATG-3' and (r) 5'-ATGGTGGTGAAGACGCCAGTA-3'. The PCR products were stained with ethidium bromide (Invitrogen) and analyzed by gel electrophoresis. All densitometry analyses were performed using ImageJ software. Signal intensities were normalized to gapdh used as housekeeping gene.

\section{Histological analysis}

Paraffin-embedded samples were cut into $5 \mu \mathrm{m}$ thick sections. Sections were deparaffined, rehydrated before antigen retrieval by 20 -min incubation at $96^{\circ} \mathrm{C}$ in citrate buffer at $\mathrm{pH} 7.3$ and nonspecific sites were blocked with $5 \%$ BSA. For immunohistochemistry (IHC), endogenous peroxidase was inhibited by incubation with $3 \% \mathrm{H}_{2} \mathrm{O}_{2}$, the slides were then successively incubated with primary antibodies, biotin-conjugated secondary antibodies and HRP-conjugated streptavidin in PBS with $0.1 \%$ Tween and $1 \%$ BSA before incubation with AEC solution (Vector Laboratories) and counterstaining with hematoxylin. Apoptotic cells were detected by TUNEL assay using the ApopTag plus Peroxidase In Situ Apoptosis Detection Kit (Millipore). For immunohistofluorescence (IHF), the slides were successively incubated with primary antibodies and fluorochrome-conjugated secondary antibodies in PBS with $0.1 \%$ Tween and $1 \%$ BSA and were finally mounted using Prolong Gold (Invitrogen). IHC and IHF images were captured on a Nikon Eclipse E400 microscope at a 40x magnification in the visible or fluorescence mode, respectively.

\section{X-Gal staining}

Tumors were fixed in PBS with $20 \%$ sucrose and embedded in OCT (Labonord). Samples were cut into 5- $\mu \mathrm{m}$ thick sections and fixed with $2 \%$ PFA before incubation with a $1 \mathrm{mg} / \mathrm{mL}$ X-Gal solution (Roche) [13] for $24 \mathrm{~h}$. Slides were then washed with PBS. Cells fixed with $2 \%$ PFA were incubated with a $1 \mathrm{mg} / \mathrm{mL} \mathrm{X-Gal}$ solution for $24 \mathrm{~h}$ and washed with PBS. Images were captured on a Zeiss primovert microscope with an AxioCam at $40 \mathrm{X}$ magnification.

\section{DDAOG staining}

Tumors were fixed in PBS with 20\% sucrose and embedded in OCT (Labonord). Samples were cut into 5- $\mu \mathrm{m}$ thick sections and fixed with 2\% PFA before incubation with a $10 \mu \mathrm{M}$ DDAOG solution (LifeTechnologies) for
60 minutes. Slides were then washed with PBS. Images were captured on a Nikon Eclipse E400 epifluorescent microscope at 40x magnification. Cells fixed with $2 \%$ PFA were incubated with a $10 \mu \mathrm{M}$ DDAOG solution for 60 minutes and washed with PBS before flow cytometry analysis (FACSCalibur, BD Biosciences).

\section{Statistical analyzes}

Experimental data are expressed as means \pm SEM. Statistical analysis was performed using R software. The Mann-Whitney $U$ test was used to compare data between two treatment groups. Differences were considered statistically significant as follows: $* P \leq 0.05$ and $* * P \leq 0.01$.

\section{ACKNOWLEDGEMENTS}

We thank Thomas Gautier (Inserm UMR866, University of burgundy, Dijon) and Sabrina Romagny (EA7269 EPHE-University of burgundy) for writing assistance and Julien Colas (Inserm UMR866, University of burgundy, Dijon) for help in microscopy analyses.

\section{GRANT SUPPORT}

This project was supported by the grants from Ligue Contre le Cancer, comités Saône et Loire and Côte d'Or. CS was supported by a grant from Conseil Régional de Bourgogne and Ligue National Contre le Cancer, AM from EPHE, and AS by a grant from INCA.

\section{REFERENCES}

1. Jemal A, Siegel R, Ward E, Hao Y, Xu J, Murray T, Thun MJ. Cancer statistics, 2008. CA Cancer J Clin. 2008; 58:71-96.

2. Segal NH, Saltz LB. Evolving treatment of advanced colon cancer. Annu Rev Med. 2009; 60:207-219.

3. Hohla F, Winder T, Greil R, Rick FG, Block NL, Schally AV. Targeted therapy in advanced metastatic colorectal cancer: current concepts and perspectives. World J Gastroenterol. 2014; 20:6102-6112.

4. Fiandalo MV, Kyprianou N. Caspase control: protagonists of cancer cell apoptosis. Exp Oncol. 2012; 34:165-175.

5. Campisi J, d'Adda di Fagagna F. Cellular senescence: when bad things happen to good cells. Nat Rev Mol Cell Biol. 2007; 8:729-740.

6. Alexandroff AB, Nicholson S, Patel PM, Jackson AM. Recent advances in bacillus Calmette-Guerin immunotherapy in bladder cancer. Immunotherapy. 2010; 2:551-560.

7. Galon J, Costes A, Sanchez-Cabo F, Kirilovsky A, Mlecnik B, Lagorce-Pagès C, Tosolini M, Camus M, 
Berger A, Wind P, Zinzindohoué F, Bruneval P, Cugnenc PH, Trajanoski Z, Fridman WH, Pagès F. Type, density, and location of immune cells within human colorectal tumors predict clinical outcome. Science. 2006; 313:1960-1964.

8. Zitvogel L, Apetoh L, Ghiringhelli F, André F, Tesniere A, Kroemer $\mathrm{G}$. The anticancer immune response: indispensable for therapeutic success? J Clin Invest. 2008; 118:1991-2001.

9. Onier N, Hilpert S, Arnould L, Saint-Giorgio V, Davies JG, Jeannin JF. Cure of colon cancer metastasis in rats with the new lipid A OM 174. Apoptosis of tumor cells and immunization of rats. Clin Exp Metastasis. 1999; 17:299-306.

10. Gautier T, Paul C, Deckert V, Desrumaux C, Klein A, Labbé J, Le Guern N, Athias A, Monier S, Hammann A, Bettaieb A, Jeannin JF, Lagrost L. Innate immune response triggered by triacyl lipid A is dependent on phospholipid transfer protein (PLTP) gene expression. FASEB J. 2010; 24:3544-3554.

11. Onier N, Hilpert S, Reveneau S, Arnould L, Saint-Giorgio V, Exbrayat JM, Jeannin JF. Expression of inducible nitric oxide synthase in tumors in relation with their regression induced by lipid A in rats. Int J Cancer. 1999; 81:755-760.

12. Isambert N, Fumoleau P, Paul C, Ferrand C, Zanetta S, Bauer J, Ragot K, Lizard G, Jeannin JF, Bardou M. Phase I study of OM-174, a lipid A analogue, with assessment of immunological response, in patients with refractory solid tumors. BMC Cancer. 2013; 13:172.

13. Debacq-Chainiaux F, Erusalimsky JD, Campisi J, Toussaint O. Protocols to detect senescence-associated beta-galactosidase (SA-betagal) activity, a biomarker of senescent cells in culture and in vivo. Nat Protoc. 2009; 4:1798-1806.

14. Glehen O, Mohamed F, Gilly FN. Peritoneal carcinomatosis from digestive tract cancer: new management by cytoreductive surgery and intraperitoneal chemohyperthermia. Lancet Oncol. 2004; 5:219-228.

15. Larmonier CB, Arnould L, Larmonier N, Baumann S, Moutet M, Saint-Giorgio V, Pance A, Jeannin JF. Kinetics of tumor cell apoptosis and immune cell activation during the regression of tumors induced by lipid A in a rat model of colon cancer. Int J Mol Med. 2004; 13:355-361.

16. Vermorken JB, Claessen AM, van Tinteren H, Gall HE, Ezinga R, Meijer S, Scheper RJ, Meijer CJ, Bloemena E, Ransom JH, Hanna MG, Pinedo HM. Active specific immunotherapy for stage II and stage III human colon cancer: a randomised trial. Lancet. 1999; 353:345-350.

17. Adams S. Toll-like receptor agonists in cancer therapy. Immunotherapy. 2009; 1:949-964.

18. Vacchelli E, Eggermont A, Sautès-Fridman C, Galon J, Zitvogel L, Kroemer G, Galluzzi L. Trial Watch: Toll-like receptor agonists for cancer therapy. Oncoimmunology. 2013; 2:e25238.

19. Chen WJ, Yuan SF, Yan QY, Xiong JP, Wang SM, Zheng WE, Zhang W, Sun HY, Chen H, Wu LL.
Intrapleural chemo- and hyperthermotherapies for malignant pleural effusion: a randomized prospective study. Cancer Invest. 2012; 30:126-130.

20. Wang X, Wong SC, Pan J, Tsao SW, Fung KH, Kwong DL, Sham JS, Nicholls JM. Evidence of cisplatin-induced senescent-like growth arrest in nasopharyngeal carcinoma cells. Cancer Res. 1998; 58:5019-5022.

21. Fang K, Chiu CC, Li CH, Chang YT, Hwang HT. Cisplatininduced senescence and growth inhibition in human non-small cell lung cancer cells with ectopic transfer of p16INK4a. Oncol Res. 2007; 16:479-488.

22. Qu K, Xu X, Liu C, Wu Q, Wei J, Meng F, Zhou L, Wang Z, Lei L, Liu P. Negative regulation of transcription factor FoxM1 by p53 enhances oxaliplatin-induced senescence in hepatocellular carcinoma. Cancer Lett. 2013; 331:105-114.

23. Haugstetter AM, Loddenkemper C, Lenze D, Gröne J, Standfuss C, Petersen I, Dörken B, Schmitt CA. Cellular senescence predicts treatment outcome in metastasised colorectal cancer. Br J Cancer. 2010; 103:505-509.

24. Berman AE, Leontieva OV, Natarajan V, McCubrey JA, Demidenko ZN, Nikiforov MA. Recent progress in genetics of aging, senescence and longevity: focusing on cancerrelated genes. Oncotarget. 2012; 3:1522-1532.

25. Leontieva OV, Natarajan V, Demidenko ZN, Burdelya LG, Gudkov AV, Blagosklonny MV. Hypoxia suppresses conversion from proliferative arrest to cellular senescence. Proc Natl Acad Sci U S A. 2012; 109:13314-13318.

26. Leontieva OV, Demidenko ZN, Blagosklonny MV. Contact inhibition and high cell density deactivate the mammalian target of rapamycin pathway, thus suppressing the senescence program. Proc Natl Acad Sci U S A. 2014; 111:8832-8837.

27. Leontieva OV, Blagosklonny MV. DNA damaging agents and p53 do not cause senescence in quiescent cells, while consecutive re-activation of mTOR is associated with conversion to senescence. Aging. 2010; 2:924-935.

28. Leelawat K, Narong S, Udomchaiprasertkul W, Leelawat S, Tungpradubkul S. Inhibition of PI3K increases oxaliplatin sensitivity in cholangiocarcinoma cells. Cancer cell international. 2009; 9:3.

29. Xue W, Zender L, Miething C, Dickins RA, Hernando E, Krizhanovsky V, Cordon-Cardo C, Lowe SW. Senescence and tumour clearance is triggered by p53 restoration in murine liver carcinomas. Nature. 2007; 445: 656-660.

30. Fridlender ZG, Sun J, Kim S, Kapoor V, Cheng G, Ling L, Worthen GS, Albelda SM. Polarization of tumor-associated neutrophil phenotype by TGF-beta: "N1" versus "N2" TAN. Cancer Cell. 2009; 16:183-194.

31. Martin F, Caignard A, Jeannin JF, Leclerc A, Martin M. Selection by trypsin of two sublines of rat colon cancer cells forming progressive or regressive tumors. Int J Cancer. $1983 ; 32: 623-627$. 